\title{
Diastereoselective and enantioselective preparation of nor-mevaldic acid surrogates through desymmetrisation methodology. Enantioselective synthesis of $(+)$ and $(-)$ nor-mevalonic lactones
}

\author{
José Manuel Botubol-Ares a , María Jesús Durán-Peña a , Rosario Hernández-Galán a , \\ Isidro G. Collado ${ }^{\mathrm{a}}$, Laurence M. Harwood ${ }^{\mathrm{b}}$, Antonio J. Macías-Sánchez ${ }^{\mathrm{a}, *}$ \\ ${ }^{a}$ Departamento de Química Orgánica, Facultad de Ciencias, Campus Universitario Puerto Real, Universidad de Cádiz, Puerto Real, Cádiz 11510, Spain \\ ${ }^{\mathrm{b}}$ Department of Chemistry, University of Reading, Whiteknights, Reading RG6 6AD, United Kingdom
}

\section{A R T I C L E I N F O}

\section{Article history:}

Received 21 July 2015

Received in revised form 2 August 2015

Accepted 3 August 2015

Available online 8 August 2015

Dedicated to Professor Steven V. Ley on the occasion of his 70th birthday

\begin{abstract}
A B S T R A C T
Solvent-free desymmetrisation of a meso-dialdehyde with chiral alcohols, led to preparation of 4silyloxy-6-alkyloxytetrahydro-2H-pyran-2-one derivatives with a $96 \%$ de. This methodology, which yields the corresponding methyl nor-mevaldates with $99 \%$ ee, has been applied to the enantioselective synthesis of the $(-)-(R)$ and $(+)-(S)$ nor-mevalonic acid lactones.
\end{abstract}

(C) 2015 Elsevier Ltd. All rights reserved.

Keywords:

Botrytis cinerea

Desymmetrisation

meso-Dialdehyde

Nor-methyl mevaldate

\section{Introduction}

Enantioselective desymmetrisation of meso or prochiral compounds has become a powerful tool in the preparation of enantiomerically pure compounds, ${ }^{1}$ using chiral reagents, enzymes ${ }^{2}$ or catalysts. ${ }^{3}$ Symmetrical or achiral precursors may provide a synthetic advantage by permitting inclusion of a certain degree of complexity before introducing chirality at the desymmetrisation step, instead of incorporating chirality at very early steps of a synthesis.

This approach has been successfully applied to the desymmetrisation of a range of substrates such as dienes, ${ }^{4}$ meso-anhydrides, ${ }^{5}$ meso-diols ${ }^{6}$ and meso-dialdehydes. Desymmetrisation of meso-dialdehydes has been performed via Horner-Wadsworth-Emmons reaction, ${ }^{7}$ alkylation, ${ }^{8}$ carbonyl-ene cyclisation reaction ${ }^{9}$ and aldol condensation, either using chiral reagents, ${ }^{10}$ an organometallic catalyst ${ }^{11}$ or organocatalysis. ${ }^{12}$

In this respect, the synthetic utility of 1,3,5-trioxygenated substrates has led to much interest in their enantiocontrolled

\footnotetext{
* Corresponding author. Tel.: +34 956 012704; fax: +34 956 016193; e-mail address: antoniojose.macias@uca.es (A.J. Macías-Sánchez).
}

preparation. $^{13}$ For instance, enantiomerically pure methyl normevaldate 1 has been used as a building block for the synthesis of bioactive molecules such as $\beta$-hydroxyacids with antifungal activity, ${ }^{14}$ HMG-CoA reductase inhibitors ${ }^{15}$ or analogues of statins ${ }^{16}$ and 6-alkyl-4-hydroxypyran-2-ones ${ }^{17}$ with oestrogenic activity. Furthermore, diverse mevalonic lactone analogues with different substituents in position 4 have been recently synthesised and reported as inhibitors of the mevalonate pathway in the bacterium Streptococcus pneumoniae. ${ }^{18}$

As part of our strategy for the design of antifungal agents against Botrytis and Colletotrichum species, ${ }^{19}$ we have recently reported the preparation of 4-hydroxy-6-(1-phenylethoxy)tetrahydro-2H-pyran-2-one (2) as a selective antifungal agent against the phytopathogen Botrytis cinerea via the desymmetrisation reaction of a suitable meso-dialdehyde precursor $\mathbf{3}$ with chiral phenylethanol. $^{20,21}$

In this paper we extend this methodology to the preparation of a range of 6-alkyloxy-4-silyloxytetrahydro- $2 H$-pyran-2-ones using different chiral alcohols. The use of the dialdehyde $\mathbf{3}$ as a building block for the enantioselective preparation of 1,3,5-trioxygenated substrates related to methyl nor-mevaldate $\mathbf{1}$, such as normevalonic acid lactones $(-)-(R)-\mathbf{4}$ and $(+)-(S)-\mathbf{5}$ is also described 
(Fig. 1). These compounds are of interest for structure-activity relationship (SAR) studies.<smiles>COC(=O)C[C@@H](O)CC=O</smiles><smiles>C[C@H](OCOC(=O)c1ccccc1)c1ccccc1</smiles><smiles>O=CCC(CC=O)O[SbH2]</smiles>

3<smiles>O=C1C[C@H](O)CCO1</smiles><smiles>O=C1C[C@H](O)CCO1</smiles>

$(-)-(R)-4$

$(+)-(S)-5$

Fig. 1. Structures $\mathbf{1}-\mathbf{5}$.

\section{Results and discussion}

\subsection{Optimisation of desymmetrisation reaction}

The absolute stereochemistries of chiral 6-arylalkyloxy-4silyloxytetrahydro-2H-pyran-2-ones (+)-6a and (+)-6b were established unequivocally in previous reports by a combination of $\mathrm{X}$-ray crystallographic analyses of the structurally related tetrahydro-2H-pyran-2-ones (+)-7a and (-)-7c, NOE difference studies and chemical correlation of tetrahydro- $2 \mathrm{H}$-pyran-2-ones $(+)-\mathbf{6 a}$ and (+)-7a with (-)-(R) methyl 3-(tert-butyldimethylsilyloxy)-5oxopentanoate (8) and of tetrahydro- $2 \mathrm{H}$-pyran-2-ones (+)-6b and (-)-7c with (+)-(S) methyl 3-(tert-butyldimethylsilyloxy)-5oxopentanoate $\mathbf{9}$ (Scheme 1). ${ }^{21}$

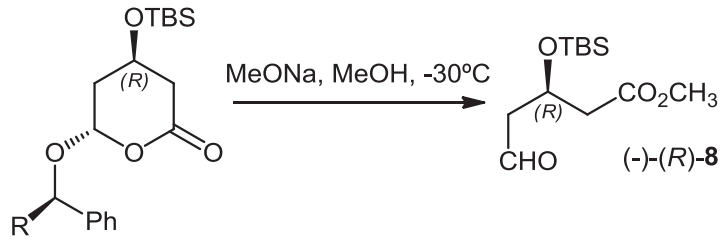

$(+)-6 \mathbf{a ~ R}=\mathrm{CH}_{3}$

$(+)-7 \mathrm{a} \mathrm{R}=\mathrm{CO}_{2} \mathrm{CH}_{3}$

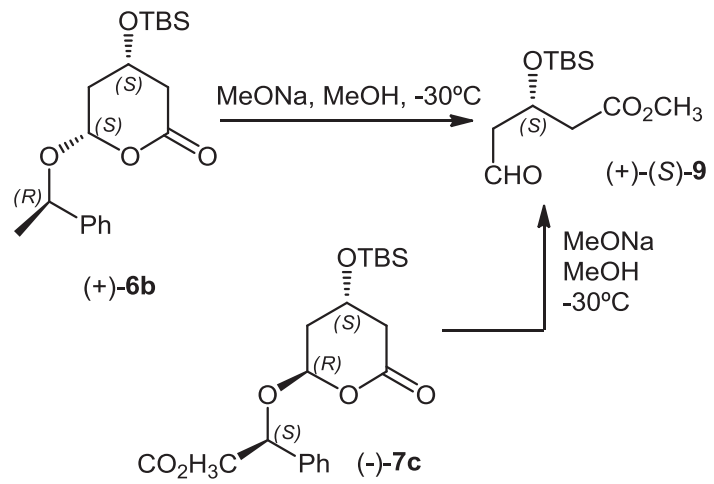

Scheme 1. Chemical correlation of lactones $(+)-6 \mathbf{a}$ and $(+)-7 \mathbf{a}$ with compound $(-)-(R)-\mathbf{8}$ and of lactones $(+)-\mathbf{6 b}$ and $(-)-\mathbf{7 c}$ with $(+)-(S)-\mathbf{9}$.

Evaluation of the influence of the chiral alcohol structure on the diastereoselectivity of the desymmetrisation process was then undertaken. Consequently, solvent-free conditions, ${ }^{20}$ which have been shown to lead to better selectivity than the use of dry THF for the preparation of lactone $(+)-\mathbf{6 a} \mathbf{a}^{21}$ were applied to a series of commercially available chiral alcohols 10-14, achieving an optimal $14.7 \%$ overall yield and $96 \%$ de with $(+)-(R)$ or $(-)-(S)-1$-(naphthalen-2-yl)ethanol (Schemes 2 and 3, Table 1, entry 6). Best yields and de were obtained when 1-arylethanols were used as chiral auxiliaries ( 6 and 14), suggesting that efficient desymmetrisation required not only a stereogenic centre directly attached to the hydroxyl group, but also a large aromatic group attached to the stereogenic centre, as use of (-)-myrtenol resulted in no asymmetric induction (Table 1 , entry 4 ).

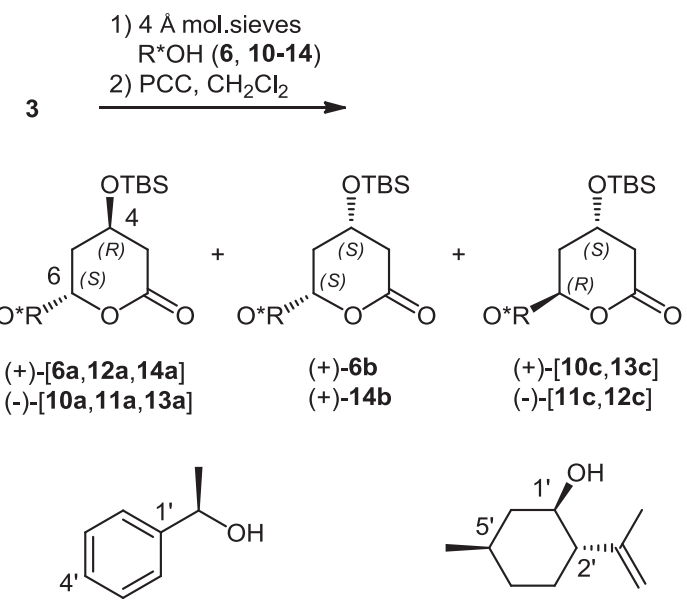

$(+)-(R)-1$-phenyl-ethanol $(6)$

(-)-(1R,2S,5R)-Isopulegol (10)

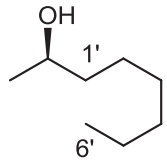

$(-)-(R)-$ octan-2-ol (11)

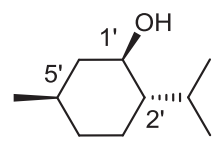

(-)-(1R,2S,5R)-menthol (13)

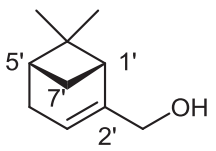

(-)-myrtenol (12)<smiles>CC(O)c1ccc2ccccc2c1</smiles>

(+)-(R)-1-(naphth-2-yl)ethanol (14)
Scheme 2. Solvent-free desymmetrisation of dialdehyde 3 with different chiral alcohols.

1) 4 Å mol. sieves, (-)-(S)-1(naphth-2-yl)ethanol (15) 2) $\mathrm{PCC}, \mathrm{CH}_{2} \mathrm{Cl}_{2}$

3<smiles>C[C@H](O[C@H]1C[C@@H]2OC(=O)C[C@]([OH+])(C1)O2)c1ccc2ccccc2c1</smiles>

$(-)-15 a(14.7 \%)$

$(-)-15 b(0.3 \%)$

Scheme 3. Preparation of (-)-15a and (-)-15b via solvent-free desymmetrisation of dialdehyde 3 with (-)-(S)-1-(naphthalen-2-yl)ethanol. 
Table 1

Solvent-free desymmetrisation of dialdehyde $\mathbf{3}$ with chiral alcohols $\mathbf{6 , 1 0}$-14

\begin{tabular}{lllc}
\hline Entry & $\mathrm{R} * \mathrm{OH}$ & Products $(\%)^{\mathrm{a}}$ & de $(\%)$ \\
\hline 1 & $(+)-(R)-\mathbf{6}$ & $\mathbf{6 a}(12.3), \mathbf{6 b}(0.6)$ & 92 \\
2 & $(-)-(1 R, 2 S, 5 R)-\mathbf{1 0}$ & $\mathbf{1 0 a}(2.5), \mathbf{1 0 c}(0.4)$ & 72 \\
3 & $(-)-(R)-\mathbf{1 1}$ & $\mathbf{1 1 a}(2.0), \mathbf{1 1 c}(0.5)$ & 60 \\
4 & $(-)-\mathbf{1 2}$ & $\mathbf{1 2 a}(2.0), \mathbf{1 2 c}(2.0)$ & 0 \\
5 & $(-)-(1 R, 2 S, 5 R)-\mathbf{1 3}$ & $\mathbf{1 3 a}(2.0), \mathbf{1 3 c}(0.6)$ & 54 \\
6 & $(+)-(R)-\mathbf{1 4}$ & $(+)-\mathbf{1 4 a}(14.7),(+)-\mathbf{1 4 b}(0.3)$ & 96 \\
\hline
\end{tabular}

a Yields obtained after chromatographic purification.

Stereochemistries of the compounds $10 \mathbf{a}-14 \mathbf{a}, 10 \mathrm{c}-13 \mathrm{c}$ and 14b were established by a combination of NOESY studies, analysis of ${ }^{1} \mathrm{H}$ NMR coupling constants and comparison with related lactones $\mathbf{6 a}, \mathbf{6 b}, \mathbf{7 a}$ and $\mathbf{7 c}$, whose absolute stereochemistries have been reported before. ${ }^{21}$ For example, compound (+)-14a presented spectroscopic data closely related to those for compound $\mathbf{6 a}$, but significantly different to those for compounds $\mathbf{6 b}$ and $\mathbf{7 c}$, while compound 14b presented spectroscopic data closely related to those for compound $\mathbf{6 b}$. Such correlations and the NOE. enhancement observed between $\mathrm{H}-6$ and $\mathrm{H}-4$ for $\mathbf{1 4 b}$ allowed us to assign their structures as $(4 R, 6 S)-4-(($ tert-butyldimethylsilyl) oxy)-6-((R)-1-(naphthalen-2-yl)ethoxy)tetrahydro-2H-pyran-2one (+)-14a and (4S,6S)-4-((tert-butyldimethylsilyl)oxy)-6- $((R)-$ 1-(naphthalen-2-yl)ethoxy)tetrahydro-2H-pyran-2-one (+)-14b, respectively $\left({ }^{1} \mathrm{H}\right.$ NMR data for compounds $6 \mathbf{a}, \mathbf{b}, 7 \mathbf{a}, \mathbf{1 0 a}-\mathbf{1 4 a}$ and 14b are presented in Tables 2 and $3 ;{ }^{1} \mathrm{H}$ NMR data for compounds 7c and 10c-13c in Table $4 ;{ }^{13} \mathrm{C}$ NMR data for compounds $\mathbf{6 a}, \mathbf{b}, \mathbf{7 a}$, $\mathbf{1 0 a}-\mathbf{1 4 a}, \mathbf{1 4 b}, \mathbf{7 c}$ and $\mathbf{1 0 c}-\mathbf{1 3} \mathbf{c}$ in Tables $S 1-S 3$ of Supplementary data).

Table 2

${ }^{1}$ H NMR spectroscopic data for $\mathbf{6 a}, \mathbf{6 b}, \mathbf{7 a}, \mathbf{1 4 a}$ and $\mathbf{1 4} \mathbf{b}$

\begin{tabular}{|c|c|c|c|c|c|}
\hline \multirow[t]{2}{*}{ Position } & \multirow{2}{*}{$\frac{\mathbf{6 \mathbf { a } ^ { 2 0 }}}{\delta_{\mathrm{H}}(\text { mult, } J \text { in } \mathrm{Hz})}$} & \multirow{2}{*}{$\frac{7 \mathbf{a}^{20}}{\delta_{\mathrm{H}}(\mathrm{mult}, J \text { in } \mathrm{Hz})}$} & \multirow{2}{*}{$\frac{14 \mathbf{a}}{\delta_{\mathrm{H}}(\text { mult, } J \text { in } \mathrm{Hz})}$} & \multirow{2}{*}{$\frac{\mathbf{6 \mathbf { b } ^ { 2 0 }}}{\delta_{\mathrm{H}}(\text { mult, } J \text { in } \mathrm{Hz})}$} & \multirow{2}{*}{$\frac{\mathbf{1 4 \mathbf { b }}}{\delta_{\mathrm{H}}(\text { mult, } J \text { in } \mathrm{Hz})}$} \\
\hline & & & & & \\
\hline \multirow[t]{2}{*}{3} & $\alpha: 2.47$ (dd, 5.6, 17.2) & $\alpha: 2.50(\mathrm{dd}, 5.6,17.2)$ & $\alpha: 2.47(\mathrm{dd}, 5.6,17.4)$ & $\alpha: 2.51(\mathrm{dd}, 9.2,16.8)$ & $\alpha: 2.51(\mathrm{dd}, 9.2,17.4)$ \\
\hline & $\beta: 2.76(\mathrm{dd}, 4.8,17.2)$ & $\beta: 2.85$ (ddd, $1.2,4.8,17.2)$ & $\beta: 2.78(\mathrm{ddd}, 0.8,4.8,17.4)$ & $\beta: 2.69(\mathrm{ddd}, 1.8,5.4,16.8)$ & $\beta: 2.69$ (ddd, $1.6,5.6,17.4)$ \\
\hline 4 & $4.33(\mathrm{~m})$ & $4.44(\mathrm{~m})$ & $4.35(\mathrm{~m})$ & 3.98, tt $(5.4,9.2)$ & $3.95(\mathrm{tt}, 5.6,9.2)$ \\
\hline \multirow[t]{2}{*}{5} & $\alpha: 1.94(\mathrm{ddd}, 3.6,5.6,13.8)$ & $\alpha: 2.13$ (dddd, 1.2, 3.8, & $\alpha 1.85(\mathrm{ddd}, 3.6,7.0,13.8)$ & $\alpha 1.82(\mathrm{ddd}, 7.7,9.2,14.0)$ & $\alpha: 1.85$ (ddd, $7.6,9.2,14.0)$ \\
\hline & $\beta: 1.85$ (ddd, $3.6,6.8,13.8)$ & $\begin{array}{l}4.8,14.0 \text { ) } \\
\beta: 1.95 \text { (ddd, } 3.8,6.8,14.0 \text { ) }\end{array}$ & $\begin{array}{l}\beta: 1.98 \text { (dddd, } 0.8,4.0 \\
5.4,13.8)\end{array}$ & $\begin{array}{l}\beta: 2.22 \text { (dddd, 1.8, 4.4, } \\
5.4,14.0 \text { ) }\end{array}$ & $\begin{array}{l}\beta: 2.21 \text { (dddd, } 1.6,4.4, \\
5.6,14.0)\end{array}$ \\
\hline 6 & $5.25(\mathrm{dd}, 3.6,5.6)$ & $5.37(\mathrm{dd}, 3.8,4.8)$ & $5.29(\mathrm{dd}, 3.6,5.4)$ & $5.04(\mathrm{dd}, 4.4,7.7)$ & $5.06(\mathrm{dd}, 4.4,7.6)$ \\
\hline $\mathrm{Si}\left(\mathrm{CH}_{3}\right)\left(\mathrm{CH}_{3}\right)$ & $0.02^{\mathrm{b}}(\mathrm{s})$ & $0.04^{\mathrm{b}}(\mathrm{s})$ & $0.00^{\mathrm{b}}(\mathrm{s})$ & $0.03^{\mathrm{b}}(\mathrm{s})$ & $0.01(\mathrm{~s})$ \\
\hline $\mathrm{Si}\left(\mathrm{CH}_{3}\right)\left(\mathrm{CH}_{3}\right)$ & $-0.03^{\mathrm{b}}(\mathrm{s})$ & $0.00^{\mathrm{b}}(\mathrm{s})$ & $-0.07^{\mathrm{b}}(\mathrm{s})$ & $0.03^{\mathrm{b}}(\mathrm{s})$ & $0.01(\mathrm{~s})$ \\
\hline $\mathrm{SiC}\left(\mathrm{CH}_{3}\right)_{3}$ & $0.76(\mathrm{~s})$ & $0.79(\mathrm{~s})$ & $0.68(s)$ & $0.86(\mathrm{~s})$ & $0.85(\mathrm{~s})$ \\
\hline $2^{\prime}$ & $7.36-7.28(\mathrm{~m})$ & $7.44-7.36(\mathrm{~m})$ & $7.50-7.42(\mathrm{~m})$ & $7.38-7.28(\mathrm{~m})$ & $7.52-7.44(\mathrm{~m})$ \\
\hline $3^{\prime}$ & $7.36-7.28(\mathrm{~m})$ & $7.44-7.36(\mathrm{~m})$ & $7.85-7.81(\mathrm{~m})$ & $7.38-7.28(\mathrm{~m})$ & $7.87-7.78(\mathrm{~m})$ \\
\hline $4^{\prime}$ & $7.36-7.28(\mathrm{~m})$ & $7.44-7.36(\mathrm{~m})$ & - & $7.38-7.28(\mathrm{~m})$ & - \\
\hline $5^{\prime}$ & $7.36-7.28(\mathrm{~m})$ & $7.44-7.36(\mathrm{~m})$ & - & $7.38-7.28(\mathrm{~m})$ & - \\
\hline $6^{\prime}$ & $7.36-7.28(\mathrm{~m})$ & $7.44-7.36(\mathrm{~m})$ & $7.74(\mathrm{~s})$ & $7.38-7.28(\mathrm{~m})$ & $7.74(\mathrm{~s})$ \\
\hline $7^{\prime}$ and $10^{\prime}$ & - & - & $7.85-7.81(\mathrm{~m})$ & - & $7.87-7.78(\mathrm{~m})$ \\
\hline $8^{\prime}$ and $9^{\prime}$ & - & - & $7.50-7.42(\mathrm{~m})$ & - & $7.52-7.44(\mathrm{~m})$ \\
\hline $\mathrm{CH}_{3} \mathrm{OCO}$ & - & $3.69(\mathrm{~s})$ & - & - & - \\
\hline $\mathrm{ArCH}\left(\mathrm{CH}_{3}\right) \mathrm{O}$ & $1.46(\mathrm{~d}, 6.6)$ & - & $1.55(\mathrm{~d}, 6.6)$ & 1.47, d (6.6) & $1.56(\mathrm{~d}, 6.4)$ \\
\hline $\mathrm{ArCHO}$ & $4.99(\mathrm{q}, 6.6)$ & $5.32(\mathrm{~s})$ & $5.15(\mathrm{q}, 6.6)$ & $5.08, \mathrm{q}(6.6)$ & $5.18(\mathrm{q}, 6.4)$ \\
\hline
\end{tabular}

a Chemical shift values, $\delta$, are in ppm, and the coupling constants, $J$, are in $\mathrm{Hz}$ (parentheses).

b Interchangeable signals.

Table 3

${ }^{1} \mathrm{H}$ NMR spectroscopic data for $\mathbf{1 0 a}-\mathbf{1 3 a}$

\begin{tabular}{|c|c|c|c|c|}
\hline \multirow[t]{2}{*}{ Position } & $10 a$ & 11a & $12 a$ & 13a \\
\hline & $\delta_{\mathrm{H}}($ mult, $J$ in $\mathrm{Hz}$ ) & $\delta_{\mathrm{H}}($ mult, $J$ in $\mathrm{Hz}$ ) & $\delta_{\mathrm{H}}($ mult, $J$ in $\mathrm{Hz})$ & $\delta_{\mathrm{H}}($ mult, $J$ in $\mathrm{Hz}$ ) \\
\hline \multirow[t]{2}{*}{3} & $\alpha: 2.35$ (dd, $6.8,17.2)$ & $\alpha: 2.48(\mathrm{dd}, 5.6,17.4)$ & $\alpha: 2.48(\mathrm{dd}, 5.6,17.6)$ & $\alpha: 2.47(\mathrm{dd}, 6.0,17.2)$ \\
\hline & $\beta: 2.77$ (ddd, $0.8,5.6$ 17.2) & $\beta: 2.76$ (ddd, $0.8,5.0,17.4)$ & $\beta: 2.75$ (ddd, $0.8,4.8,17.6)$ & $\beta: 2.76$ (ddd, $0.8,5.2,17.2$ ) \\
\hline 4 & $4.30(\mathrm{~m})$ & $4.35(\mathrm{~m})$ & $4.34(\mathrm{~m})$ & $4.36(\mathrm{~m})$ \\
\hline \multirow[t]{2}{*}{5} & $\alpha: 1.79(\mathrm{ddd}, 4.0,8.4,13.6)$ & $\alpha: 2.01-1.90(\mathrm{~m})$ & $\alpha: 1.99-1.90(\mathrm{~m})$ & $\alpha: 1.95-1.85(\mathrm{~m})$ \\
\hline & $\beta: 2.06-1.91(\mathrm{~m})$ & $\beta: 2.01-1.90(\mathrm{~m})$ & $\beta: 1.99-1.90(\mathrm{~m})$ & $\beta: 1.95-1.85(\mathrm{~m})$ \\
\hline 6 & $5.50(\mathrm{t}, 4.0)$ & $5.54(\mathrm{dd}, 3.8,5.0)$ & $5.45(\mathrm{dd}, 4.0,4.8)$ & $5.62(\mathrm{t}, 4.2)$ \\
\hline $\mathrm{Si}\left(\mathrm{CH}_{3}\right)\left(\mathrm{CH}_{3}\right)$ & $0.00(\mathrm{~s})$ & $0.07(\mathrm{~s})$ & $0.06(\mathrm{~s})$ & $0.07(\mathrm{~s})$ \\
\hline $\mathrm{Si}\left(\mathrm{CH}_{3}\right)\left(\mathrm{CH}_{3}\right)$ & $0.00(\mathrm{~s})$ & $0.07(\mathrm{~s})$ & $0.06(\mathrm{~s})$ & $0.07(\mathrm{~s})$ \\
\hline $\mathrm{SiC}\left(\mathrm{CH}_{3}\right)_{3}$ & $0.82(\mathrm{~s})$ & $0.88-0.85(\mathrm{~m})$ & $0.86(\mathrm{~s})$ & $0.89-0.70(\mathrm{~m})$ \\
\hline $\mathrm{CH}(\mathrm{H}) \mathrm{O}$ & - & - & $4.15(\mathrm{dd}, 1.4,12.2)$ & - \\
\hline $\mathrm{CH}(\mathrm{H}) \mathrm{O}$ & - & - & $4.05(\mathrm{dd}, 1.4,12.2)$ & - \\
\hline $\mathrm{CH}\left(\mathrm{CH}_{3}\right) \mathrm{O}$ & - & 3.92 (sext, 6.4) & - & - \\
\hline $\mathrm{CH}\left(\mathrm{CH}_{3}\right) \mathrm{O}$ & - & $1.13(\mathrm{~d}, 6.4)$ & - & - \\
\hline \multirow[t]{2}{*}{$1^{\prime}$} & $3.68(\mathrm{dt}, 4.2,10.8)$ & $\alpha: 1.44-1.36(\mathrm{~m})$ & $2.14-2.08(\mathrm{~m})$ & $3.64(\mathrm{dt}, 4.4,10.6)$ \\
\hline & & $\beta: 1.54-1.48(\mathrm{~m})$ & & \\
\hline $2^{\prime}$ & $2.06-1.91(\mathrm{~m})$ & $1.32-1.20(\mathrm{~m})$ & - & $1.24-1.14(\mathrm{~m})$ \\
\hline \multirow[t]{2}{*}{$3^{\prime}$} & $\alpha: 1.48-1.24(\mathrm{~m})$ & $1.32-1.20(\mathrm{~m})$ & $5.54(\mathrm{~m})$ & $\alpha: 1.02-0.92(\mathrm{~m})$ \\
\hline & $\beta: 1.64-1.54(\mathrm{~m})$ & & & $\beta: 1.68-1.54(\mathrm{~m})$ \\
\hline \multirow[t]{2}{*}{$4^{\prime}$} & $1.48-1.24(\mathrm{~m})$ & $1.32-1.20(\mathrm{~m})$ & $2.27(\mathrm{~m})$ & $\alpha: 0.89-0.70(\mathrm{~m})$ \\
\hline & & & & $\beta: 1.68-1.54(\mathrm{~m})$ \\
\hline $5^{\prime}$ & $1.64-1.54(\mathrm{~m})$ & $1.32-1.20(\mathrm{~m})$ & $2.14-2.08(\mathrm{~m})$ & $1.40-1.28(\mathrm{~m})$ \\
\hline
\end{tabular}


Table 3 (continued)

\begin{tabular}{|c|c|c|c|c|}
\hline \multirow[t]{2}{*}{ Position } & $10 a$ & 11a & $12 a$ & 13a \\
\hline & $\delta_{\mathrm{H}}($ mult, $J$ in $\mathrm{Hz})$ & $\delta_{\mathrm{H}}($ mult, $J$ in $\mathrm{Hz})$ & $\delta_{\mathrm{H}}($ mult, $J$ in $\mathrm{Hz})$ & $\delta_{\mathrm{H}}($ mult, $J$ in $\mathrm{Hz}$ ) \\
\hline $6^{\prime}$ & $\begin{array}{l}\alpha: 0.84-0.78(\mathrm{~m}) \\
\beta: 2.06-1.91(\mathrm{~m})\end{array}$ & $0.88-0.85(\mathrm{~m})$ & - & $\begin{array}{l}\alpha: 0.89-0.70(\mathrm{~m}) \\
\beta: 2.03-1.96(\mathrm{~m})\end{array}$ \\
\hline $7^{\prime}$ & - & - & $\begin{array}{l}\alpha: 1.12(\mathrm{~d}, 8.8) \\
\beta: 2.39(\mathrm{dt}, 5.6,8.8)\end{array}$ & - \\
\hline $\mathrm{C}^{\prime} \mathrm{C}\left(\mathrm{CH}_{3}\right)=\mathrm{C}(\mathrm{H})(\mathrm{H})$ & $1.60(\mathrm{~s})$ & - & - & - \\
\hline $\mathrm{C}^{\prime} \mathrm{C}\left(\mathrm{CH}_{3}\right)=\mathrm{C}(\mathrm{H})(\mathrm{H})$ & 4.66 (br s) & - & - & - \\
\hline $\mathrm{C}^{\prime} \mathrm{C}\left(\mathrm{CH}_{3}\right)=\mathrm{C}(\mathrm{H})(\mathrm{H})$ & 4.67 (br s) & - & - & - \\
\hline $\mathrm{C} 2^{\prime} \mathrm{CH}\left(\mathrm{CH}_{3}\right)\left(\mathrm{CH}_{3}\right)$ & - & - & - & $0.89-0.70(\mathrm{~m})$ \\
\hline $\mathrm{C}^{\prime} \mathrm{CH}\left(\mathrm{CH}_{3}\right)\left(\mathrm{CH}_{3}\right)$ & - & - & - & $0.78(\mathrm{~d}, 6.8)$ \\
\hline $\mathrm{C}^{\prime}-\left(\mathrm{CH}_{3}\right)\left(\mathrm{CH}_{3}\right)$ & - & - & $0.81^{\mathrm{b}}(\mathrm{s})$ & - \\
\hline $\mathrm{C}^{\prime}-\left(\mathrm{CH}_{3}\right)\left(\mathrm{CH}_{3}\right)$ & - & - & $1.28^{\mathrm{b}}(\mathrm{s})$ & - \\
\hline $\mathrm{C}^{\prime}-\mathrm{CH}_{3}$ & $0.89(\mathrm{~d}, 6.8)$ & - & - & $0.88(\mathrm{~d}, 6.8)$ \\
\hline
\end{tabular}

${ }^{a}$ Chemical shift values, $\delta$, are in ppm, and the coupling constants, $J$, are in $\mathrm{Hz}$ (parentheses).

b Interchangeable signals.

Table 4

${ }^{1} \mathrm{H}$ NMR spectroscopic data for $\mathbf{7 c}$ and $\mathbf{1 0 c}-\mathbf{1 3} \mathbf{c}^{\mathrm{a}}$

\begin{tabular}{|c|c|c|c|c|c|}
\hline \multirow[t]{2}{*}{ Position } & \multirow{2}{*}{$\frac{7 \mathbf{c}^{20}}{\delta_{\mathrm{H}}(\text { mult, } J \text { in } \mathrm{Hz})}$} & \multirow{2}{*}{$\frac{\mathbf{1 0 c}}{\delta_{\mathrm{H}}(\text { mult, } J \text { in } \mathrm{Hz})}$} & \multirow{2}{*}{$\frac{11 c}{\delta_{\mathrm{H}}(\text { mult, } J \text { in } \mathrm{Hz})}$} & \multirow{2}{*}{$\frac{12 \mathrm{c}}{\delta_{\mathrm{H}}(\text { mult, } J \text { in } \mathrm{Hz})}$} & \multirow{2}{*}{$\frac{13 c}{\delta_{\mathrm{H}}(\text { mult, } J \text { in } \mathrm{Hz})}$} \\
\hline & & & & & \\
\hline 3 & $\alpha: 2.49(\mathrm{dd}, 4.8,17.3)$ & $\alpha: 2.45(\mathrm{dd}, 5.2,17.2)$ & $\alpha: 2.48(\mathrm{dd}, 5.2,17.2)$ & $\alpha: 2.48(\mathrm{dd}, 5.2,17.2)$ & $\alpha: 2.48(\mathrm{dd}, 5.0,17.2)$ \\
\hline & $\beta: 2.70(\mathrm{dd}, 4.8,17.3)$ & $\beta: 2.69(\mathrm{dd}, 5.2,17.2)$ & $\beta: 2.74(\mathrm{dd}, 5.2,17.2)$ & $\beta: 2.75(\mathrm{dd}, 5.2,17.2)$ & $\beta: 2.73(\mathrm{dd}, 5.0,17.2)$ \\
\hline 4 & 4.36 (quint, 4.8) & 4.29 (quint, 5.2) & 4.33 (quint, 5.2) & 4.34 (quint, 5.2) & 4.33 (quint, 5.0) \\
\hline 5 & $2.16(t, 4.8)$ & $1.91-1.87(\mathrm{~m})$ & $1.96(\mathrm{~m})$ & $1.98-1.94(\mathrm{~m})$ & $2.04-1.94(\mathrm{~m})$ \\
\hline 6 & $5.64(\mathrm{t}, 4.6)$ & $5.42(\mathrm{dd}, 4.2,5.2)$ & $5.52(\mathrm{dd}, 4.6,5.2)$ & $5.46(t, 4.4)$ & $5.50(t, 5.0)$ \\
\hline $\mathrm{Si}\left(\mathrm{CH}_{3}\right)\left(\mathrm{CH}_{3}\right)$ & $0.08^{\mathrm{b}}(\mathrm{s})$ & $0.05(\mathrm{~s})$ & $0.07(\mathrm{~s})$ & $0.06(\mathrm{~s})$ & $0.07(\mathrm{~s})$ \\
\hline $\mathrm{Si}\left(\mathrm{CH}_{3}\right)\left(\mathrm{CH}_{3}\right)$ & $0.07^{\mathrm{b}}(\mathrm{s})$ & $0.05(\mathrm{~s})$ & $0.07(\mathrm{~s})$ & $0.06(\mathrm{~s})$ & $0.07(\mathrm{~s})$ \\
\hline $\mathrm{SiC}\left(\mathrm{CH}_{3}\right)_{3}$ & $0.87(\mathrm{~s})$ & $0.86(s)$ & $0.90-0.85(\mathrm{~m})$ & $0.86(\mathrm{~s})$ & $0.91-0.80(\mathrm{~m})$ \\
\hline $\mathrm{CH}(\mathrm{H}) \mathrm{O}$ & - & - & - & $4.20(\mathrm{dd}, 1.6,12.8)$ & - \\
\hline $\mathrm{CH}(\mathrm{H}) \mathrm{O}$ & - & - & - & $4.17(\mathrm{dd}, 1.6,12.8)$ & - \\
\hline $\mathrm{CH}\left(\mathrm{CH}_{3}\right) \mathrm{O}$ & - & - & 3.83 (sext, 6.0) & - & - \\
\hline $\mathrm{CH}\left(\mathrm{CH}_{3}\right) \mathrm{O}$ & - & - & $1.21(\mathrm{~d}, 6.4)$ & - & - \\
\hline $\mathrm{ArCHO}$ & $5.47(\mathrm{~s})$ & - & - & - & - \\
\hline $1^{\prime}$ & - & $3.60(\mathrm{dt}, 4.4,10.2)$ & $\begin{array}{l}\alpha: 1.44-1.36(\mathrm{~m}) \\
\beta: 1.54-1.46(\mathrm{~m})\end{array}$ & - & $3.45(\mathrm{dt}, 4.8,10.8)$ \\
\hline $2^{\prime}$ & $7.44-7.32(\mathrm{~m})$ & $1.96(\mathrm{ddd}, 3.6,10.2,12.8)$ & $1.30-1.24(\mathrm{~m})$ & $2.12-2.08(\mathrm{~m})$ & $1.26-1.18(\mathrm{~m})$ \\
\hline $3^{\prime}$ & $7.44-7.32(\mathrm{~m})$ & $\begin{array}{l}\alpha: 1.32(\mathrm{~m}) \\
\beta: 1.66-1.57(\mathrm{~m})\end{array}$ & $1.30-1.24(\mathrm{~m})$ & $5.52(\mathrm{~m})$ & $\begin{array}{l}\alpha: 1.05-0.92(\mathrm{~m}) \\
\beta: 1.65-1.58(\mathrm{~m})\end{array}$ \\
\hline $4^{\prime}$ & $7.44-7.32(\mathrm{~m})$ & $\begin{array}{l}\alpha: 0.88-0.85(\mathrm{~m}) \\
\beta: 1.66-1.57(\mathrm{~m})\end{array}$ & $1.30-1.24(\mathrm{~m})$ & $2.27(\mathrm{~m})$ & $\begin{array}{l}\alpha: 0.91-0.80(\mathrm{~m}) \\
\beta: 1.65-1.58(\mathrm{~m})\end{array}$ \\
\hline $5^{\prime}$ & $7.44-7.32(\mathrm{~m})$ & $1.50-1.41(\mathrm{~m})$ & $1.30-1.24(\mathrm{~m})$ & $2.12-2.08(\mathrm{~m})$ & $1.42-1.33(\mathrm{~m})$ \\
\hline $6^{\prime}$ & $7.44-7.32(\mathrm{~m})$ & $\begin{array}{l}\alpha: 1.02(\mathrm{~m}) \\
\beta: 2.14-2.09(\mathrm{~m})\end{array}$ & $0.90-0.85(\mathrm{~m})$ & - & $\begin{array}{l}\alpha: 1.05-0.92(\mathrm{~m}) \\
\beta: 2.22-2.16(\mathrm{~m})\end{array}$ \\
\hline $7^{\prime}$ & - & - & - & $\begin{array}{l}\alpha: 1.15(\mathrm{~d}, 8.8) \\
\beta: 2.38(\mathrm{dt}, 5.6,8.8)\end{array}$ & - \\
\hline $\mathrm{C} 2^{\prime} \mathrm{C}\left(\mathrm{CH}_{3}\right)=\mathrm{CH}_{2}$ & - & $1.70(\mathrm{~s})$ & - & - & - \\
\hline $\mathrm{C}^{\prime} \mathrm{C}\left(\mathrm{CH}_{3}\right)=\mathrm{C}(H)(\mathrm{H})$ & - & 4.76 (br s) & - & - & - \\
\hline $\mathrm{C}^{\prime} \mathrm{C}\left(\mathrm{CH}_{3}\right)=\mathrm{C}(\mathrm{H})(\mathrm{H})$ & - & 4.78 (br s) & - & - & - \\
\hline $\mathrm{C}^{\prime} \mathrm{CH}\left(\mathrm{CH}_{3}\right)\left(\mathrm{CH}_{3}\right)$ & - & - & - & - & $0.91-0.80(\mathrm{~m})$ \\
\hline $\mathrm{C}^{\prime} \mathrm{CH}\left(\mathrm{CH}_{3}\right)\left(\mathrm{CH}_{3}\right)$ & - & - & - & - & $0.77(\mathrm{~d}, 7.2)$ \\
\hline $\mathrm{C}^{\prime} \mathrm{CH}\left(\mathrm{CH}_{3}\right)\left(\mathrm{CH}_{3}\right)$ & - & - & - & - & $2.04-1.94(\mathrm{~m})$ \\
\hline $\mathrm{C}^{\prime}-\left(\mathrm{CH}_{3}\right)\left(\mathrm{CH}_{3}\right)$ & - & - & - & $1.26^{\mathrm{b}}(\mathrm{s})$ & - \\
\hline $\mathrm{C}^{\prime}-\left(\mathrm{CH}_{3}\right)\left(\mathrm{CH}_{3}\right)$ & - & - & - & $0.81^{\mathrm{b}}(\mathrm{s})$ & - \\
\hline $\mathrm{C}^{\prime}-\mathrm{CH}_{3}$ & - & $0.90(\mathrm{~d}, 6.4)$ & - & - & $0.91-0.80(\mathrm{~m})$ \\
\hline $\mathrm{CH}_{3} \mathrm{COO}$ & $3.70(\mathrm{~s})$ & - & - & - & - \\
\hline
\end{tabular}

a Chemical shift values, $\delta$, are in ppm, and the coupling constants, $J$, are in $\mathrm{Hz}$ (parentheses).

b Interchangeable signals.

\subsection{Preparation of nor-mevalonic acid lactones $(-)-(R)-4$ and $(+)-(S)-5$}

To exemplify the utility of our methodology, and in order to prepare reference compounds for SAR studies, ${ }^{18}$ an enantioselective synthesis of nor-mevalonic acid lactones $(-)-(R)-\mathbf{4}$ and $(+)-(S)-$ $\mathbf{5}^{13 e, 22}$ was undertaken from lactones $(+)-\mathbf{1 4 a}$ and $(-)-\mathbf{1 5 a}$ (Scheme 4 ), which were in turn obtained from dialdehyde $\mathbf{3}$ (Schemes 2 and $3)$. Treatment of lactone $(+)-\mathbf{1 4 a}$ with $\mathrm{MeONa}$ and $\mathrm{MeOH}$ at $-35^{\circ} \mathrm{C}$ yielded $(-)-(R)-8$ in $52 \%$ yield and $99 \%$ ee. ${ }^{23}$ Reduction of $(-)-(R)-8$ with $\mathrm{NaBH}_{4}$ at $-35{ }^{\circ} \mathrm{C}$ to yield (-)-(R)-16 ${ }^{24}$ and subsequent lactonisation with PTSA $^{25}$ produced the corresponding lactone $(-)-(R)-$ 17 that was subsequently treated with $\mathrm{TBAF} / \mathrm{AcOH},{ }^{26}$ affording $(R)-$ nor-mevalonic acid lactone $((-)-(R)-4)$ in $70 \%$ yield and $79 \%$ ee..$^{23,27}$ Desymmetrisation of the dialdehyde 3 with (-)-(S)-1-(naphthalen2-yl)ethanol afforded the corresponding lactone (-)-15a in $14.7 \%$ overall yield and $96 \%$ de (Scheme 3 ); methanolysis of which, reduction with $\mathrm{NaBH}_{4}$, lactonisation ${ }^{25}$ to yield (+)-(S)-19 and deprotection with TBAF/AcOH, ${ }^{25}$ yielded $(S)$-nor-mevalonic acid lactone $((+)-(S)-5)$ in $67 \%$ overall yield and $96 \%$ ee. $^{23}$ 


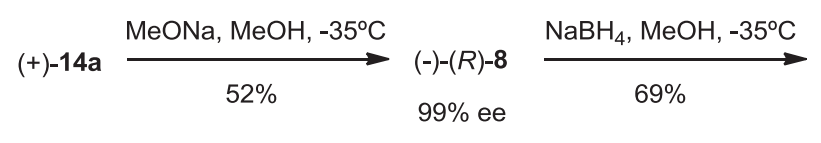<smiles>[R20]C1CCOC(=O)C1</smiles>

$(-)-(R)-16$

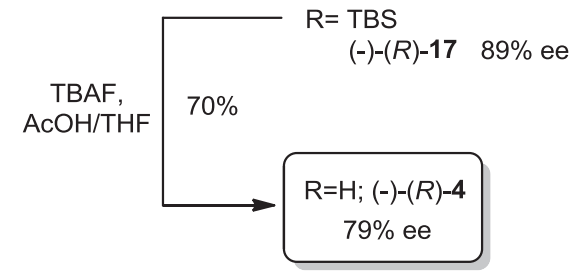

$\underset{(-)-15 a}{\stackrel{\mathrm{MeONa}, \mathrm{MeOH},-35^{\circ} \mathrm{C}}{\longrightarrow}} \underset{90 \%}{(+)-(S)-9} \frac{\mathrm{NaBH}_{4}, \mathrm{MeOH},-35^{\circ} \mathrm{C}}{72 \% \text { ee }}$

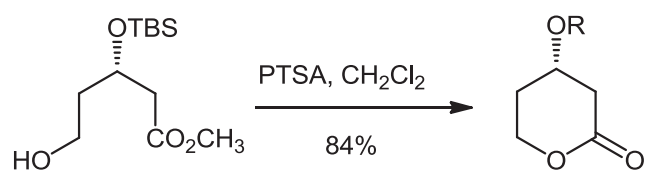

$(+)-(S)-18$

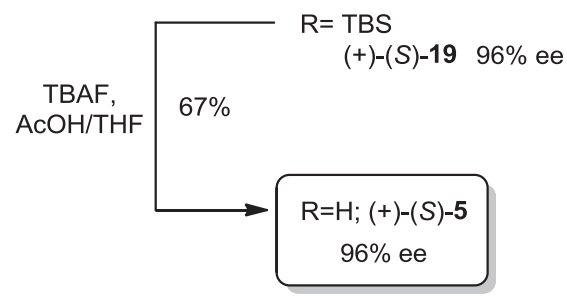

Scheme 4. Preparation of $(R)$ and $(S)$ nor-mevalonic acid lactones $((-)-(R)-\mathbf{4})$ and $((+)-(S)-5)$.

\section{Conclusions}

In summary, the optimization of the desymmetrisation of meso dialdehyde 3 led to the selective preparation of $(+)-(4 R, 6 S)-4-$ ((tert-butyldimethylsilyl)oxy)-6-((R)-1-(naphthalen-2-yl)ethoxy) tetrahydro-2H-pyran-2-one (14a) and (4S,6R)-4-((tert-butyldimethylsilyl)oxy)-6-((S)-1-(naphthalen-2-yl)ethoxy)tetrahydro-2Hpyran-2-one-(15a) in 96\% de when either $(R)-(+)-1$-(naphthalen-2yl)ethanol or $(S)-(-)-1-($ naphthalen-2-yl)ethanol was used under solvent-free conditions at room temperature. HPLC purification and methanolysis of $(+)-\left(4 R, 6 S, 1^{\prime} R\right)-(\mathbf{1 4 a})$ or $(-)-\left(4 S, 6 R, 1^{\prime} S\right)-(\mathbf{1 5 a})$ yielded (-)-(R)-methyl 3-(tert-butyldimethylsilyloxy)-5-oxopentanoate $(-)-(R)-(8)$ and $(+)-(S)$-methyl 3-(tert-butyldimethylsilyloxy)-5-oxopentanoate $(+)-(S)-(\mathbf{9})$, respectively, in $99 \%$ ee. Further transformation of these substrates allowed the enantioselective preparation of the nor-mevalonic acid lactones (-)-(R)-4 and $(+)-(S)-5$ (in $42 \%$ overall yield and $79 \%$ ee and in $41 \%$ overall yield and $96 \%$ ee, respectively).

\section{Experimental section}

\subsection{General}

Unless otherwise noted, materials and reagents were obtained from commercial suppliers and were used without further purification. Dichloromethane was freshly distilled from $\mathrm{CaH}_{2}$ and tetrahydrofuran was dried over sodium and benzophenone and freshly distilled before use. Air- and moisture-sensitive reactions were performed under an argon atmosphere. Purification by semipreparative and analytical HPLC was performed, respectively, with $250 \mathrm{~mm} \times 10 \mathrm{~mm}(10 \mu \mathrm{m}$ particles $)$ and $250 \mathrm{~mm} \times 4 \mathrm{~mm}(5 \mu \mathrm{m}$ particles) columns using a differential refractometer detector. Silica gel was used for column chromatography. TLC analyses were performed on aluminium plates coated with silica gel with fluorescent indicator (254 nm), $0.25 \mathrm{~mm}$ thick. Enantiomeric excesses (ee) were measured by GC using a Cyclosil B chiral column $(30 \mathrm{~m}$ length $\times 0.25 \mathrm{~mm}$ ID, $0.25 \mu \mathrm{m}$ film thickness), using an FID detector at $320^{\circ} \mathrm{C}$, a split injector $\left(15: 1\right.$ ratio) at $250{ }^{\circ} \mathrm{C}$, hydrogen as carrier at $10 \mathrm{psi}$ and $1 \mu \mathrm{L}$ of injection volume. Temperature program is described in the experimental section for every relevant compound. Specific rotations were determined with a digital polarimeter. Infrared spectra were recorded on an FTIR spectrophotometer and peak position reported in wavenumbers $\left(\mathrm{cm}^{-1}\right) .{ }^{1} \mathrm{H}$ spectra were recorded on spectrometers operating at 300 and $400 \mathrm{MHz} .{ }^{13} \mathrm{C}$ NMR spectra were measured at 75 and $100 \mathrm{MHz}$ with complete proton decoupling. Chemical shifts were referenced to $\mathrm{CDCl}_{3}\left(\delta_{\mathrm{H}}\right.$ $\left.7.25, \delta_{C} 77.0\right)$. NMR assignments were made by combination of $1 \mathrm{D}$ and 2D techniques. Multiplicities are described using the following abbreviations: $\mathrm{s}=$ singlet, $\mathrm{d}=$ doublet, $\mathrm{t}=$ triplet, $\mathrm{q}=$ quartet; quint=quintet; sext=sextet; $\mathrm{m}=$ multiplet, $\mathrm{br}=$ broad. Highresolution mass spectrometry (HRMS) was recorded with a double-focussing magnetic sector mass spectrometer in positive ion mode, or in a QTOF mass spectrometer in positive ion electrospray mode at $20 \mathrm{~V}$ cone voltage.

\subsection{Procedures}

4.2.1. General procedure for the preparation of 6-alkyloxy-4-(tertbutyldimethylsilyloxy)tetrahydro-2H-pyran-2-ones (6a,b, 10a-15a, 14b, 15b and 10c-13c): desymmetrisation of 3-(tert-butyldimethylsilyloxy)pentanedial (3) under solvent-free conditions followed by PCC oxidation. The requisite alcohol (see Schemes 2 and 3 of the manuscript) (2.9 mmol) was added to a mixture of 3-(tert-butyldimethylsilyloxy)pentanedial (3) $(1 \mathrm{mmol})^{20}$ and $4 \AA$ molecular sieves ( $0.5 \mathrm{~g}$ for each $\mathrm{mmol}$ of $\mathbf{3})$ under an argon atmosphere and the mixture stirred for $24 \mathrm{~h}$. The slurry was dissolved in $\mathrm{CH}_{2} \mathrm{Cl}_{2}$ $(20 \mathrm{~mL})$ and added dropwise to a suspension of PCC (3.5 mmol) and powdered molecular sieves $4 \AA$ (twice the weight of the alcohol) in dichloromethane $(70 \mathrm{~mL})$ at room temperature. The reaction mixture was stirred vigorously for $18 \mathrm{~h}$, diethyl ether was then added $(200 \mathrm{~mL})$ and the mixture was stirred for a further $1 \mathrm{~h}$. The suspension was filtered through a pad of silica gel and washed through with a further quantity of ether $(200 \mathrm{~mL})$. The ether was removed under reduced pressure to give the crude mixture of tetrahydro$2 \mathrm{H}$-pyran-2-ones, which was purified by column chromatography (petroleum ether $/ \mathrm{Et}_{2} \mathrm{O}, 90: 10$ ), to yield the corresponding tetrahydro-2H-pyran-2-ones in the ratios and yields shown below and in Table 1 and Schemes 2 and 3 of the manuscript.

4.2.1.1. (-)-(4R(S),6S(R))-4-((tert-Butyldimethylsilyl)oxy)-6(((1R,2S,5R)-5-methyl-2-(prop-1-en-2-yl)cyclohexyl)oxy)tetrahydro$2 \mathrm{H}$-pyran-2-one (10a). Yield $9.5 \mathrm{mg}, 2.5 \%$. Colourless oil; HPLC $t_{\mathrm{R}}=21 \mathrm{~min}$ (petroleum ether/ethyl acetate 93:7; flow $=3.0 \mathrm{~mL} / \mathrm{min}$ ); $[\alpha]_{\mathrm{D}}^{20}-73.7$ (c 0.1, $\mathrm{CHCl}_{3}$ ); IR (film) $\nu_{\max } 3073,2932,2858,1749$, $1644,1453,1378,1228,1145,1024,889,837,781 \mathrm{~cm}^{-1} ;{ }^{1} \mathrm{H}$ NMR $\left(\mathrm{CDCl}_{3}, 400 \mathrm{MHz}\right)$ (see Table 3); ${ }^{13} \mathrm{C} \mathrm{NMR}\left(\mathrm{CDCl}_{3}, 100 \mathrm{MHz}\right)$ (see Table S2); HRMS $\left(\mathrm{Cl}^{+}\right): m / z[\mathrm{M}+\mathrm{H}]^{+}$, found 383.2608. $\mathrm{C}_{21} \mathrm{H}_{39} \mathrm{O}_{4} \mathrm{Si}$ requires 383.2618 .

4.2.1.2. (+)-(4S(R),6R(S))-4-((tert-Butyldimethylsilyl)oxy)-6(((1R,2S,5R)-5-methyl-2-(prop-1-en-2-yl)cyclohexyl)oxy)tetrahydro$2 \mathrm{H}$-pyran-2-one $(\mathbf{1 0 c})$. Yield $1.5 \mathrm{mg}, 0.4 \%$. Colourless oil; HPLC $t_{\mathrm{R}}=26 \mathrm{~min}$ (petroleum ether/ethyl acetate 93:7; flow=3.0 $\mathrm{mL} / \mathrm{min}$ ); 
$[\alpha]_{\mathrm{D}}^{20}+44.8\left(\mathrm{c} 0.5, \mathrm{CHCl}_{3}\right)$; IR (film) $\nu_{\max } 3074,2928,2858,1750$, 1647, 1458, 1378, 1256, 1153, 1006, 837, $778 \mathrm{~cm}^{-1}$; ${ }^{1} \mathrm{H}$ NMR (400 MHz, $\mathrm{CDCl}_{3}$ ) (see Table 4); ${ }^{13} \mathrm{C}$ NMR $\left(100 \mathrm{MHz}, \mathrm{CDCl}_{3}\right.$ ) (see Table S3); HRMS $\left(\mathrm{CI}^{+}\right): m / z[\mathrm{M}+\mathrm{H}]^{+}$, found 383.2605. $\mathrm{C}_{21} \mathrm{H}_{39} \mathrm{O}_{4} \mathrm{Si}$ requires 383.2618 .

4.2.1.3. (-)-(4R(S),6S(R))-4-((tert-Butyldimethylsilyl)oxy)-6-((R)octan-2-yloxy)tetrahydro-2H-pyran-2-one (11a). Yield $7.2 \mathrm{mg}, 2 \%$. Colourless oil; HPLC $t_{\mathrm{R}}=19$ min (petroleum ether/ethyl 93:7; flow=3.0 mL/min); $[\alpha]_{\mathrm{D}}^{0}-44.9\left(\right.$ c $\left.0.1, \mathrm{CHCl}_{3}\right)$; IR (film) $\nu_{\max } 2930$, 2858, 1751, 1464, 1256, 1103, 1008, 837, $778 \mathrm{~cm}^{-1} ;{ }^{1} \mathrm{H} \mathrm{NMR}$ $\left(400 \mathrm{MHz}, \mathrm{CDCl}_{3}\right.$ ) (see Table 3$) ;{ }^{13} \mathrm{C}$ NMR $\left(100 \mathrm{MHz}, \mathrm{CDCl}_{3}\right.$ ) (see Table S2); HRMS $\left(\mathrm{CI}^{+}\right): m / z[\mathrm{M}+\mathrm{H}]^{+}$, found 359.2625. $\mathrm{C}_{19} \mathrm{H}_{39} \mathrm{O}_{4} \mathrm{Si}$ requires 359.2618 .

4.2.1.4. (-)-(4S(R),6R(S))-4-((tert-butyldimethylsilyl)oxy)-6-((R)octan-2-yloxy)tetrahydro-2H-pyran-2-one (11c). Yield $1.8 \mathrm{mg}, 0.5 \%$. Colourless oil; HPLC $t_{\mathrm{R}}=27 \mathrm{~min}$ (petroleum ether/ethyl acetate 93:7; flow=3.0 mL/min); $[\alpha]_{\mathrm{D}}^{20}-28.3\left(\right.$ c $0.2, \mathrm{CHCl}_{3}$ ); IR (film) $\nu_{\max }$ 2932, 2857, 1748, 1469, 1256, 1103, 1021, 838, $778 \mathrm{~cm}^{-1}$; ${ }^{1} \mathrm{H}$ NMR (400 $\mathrm{MHz}, \mathrm{CDCl}_{3}$ ) (see Table 4); ${ }^{13} \mathrm{C}$ NMR (100 MHz, $\mathrm{CDCl}_{3}$ ) (see Table S3); HRMS $\left(\mathrm{CI}^{+}\right): m / z[\mathrm{M}+\mathrm{H}]^{+}$, found 359.2625. $\mathrm{C}_{19} \mathrm{H}_{39} \mathrm{O}_{4} \mathrm{Si}$ requires 359.2618 .

4.2.1.5. (+)-(4R(S),6S(R))-4-((tert-Butyldimethylsilyl)oxy)-6$(((1 R, 5 S)-6,6$-dimethylbicyclo[3.1.1]hept-2-en-2-yl)methoxy)tetrahydro-2H-pyran-2-one (12a). Yield $7.6 \mathrm{mg}, 2 \%$. Colourless oil; HPLC $t_{\mathrm{R}}=40 \mathrm{~min}$ (petroleum ether/ethyl acetate $94: 6$; flow $=0.8 \mathrm{~mL} / \mathrm{min}$ ); $[\alpha]_{\mathrm{D}}^{20}+40.0\left(\right.$ c $\left.0.2, \mathrm{CHCl}_{3}\right)$; IR (film) $\nu_{\max } 2929,2858,1753,1472$, $1383,1225,1102,1030,838,778 \mathrm{~cm}^{-1} ;{ }^{1} \mathrm{H}$ NMR (400 MHz, $\mathrm{CDCl}_{3}$ ) (see Table 3); ${ }^{13} \mathrm{C}$ NMR (100 MHz, $\mathrm{CDCl}_{3}$ ) (see Table S2); HRMS $\left(\mathrm{Cl}^{+}\right): m / z[\mathrm{M}+\mathrm{H}]^{+}$, found 381.2455. $\mathrm{C}_{21} \mathrm{H}_{37} \mathrm{O}_{4} \mathrm{Si}$ requires 381.2461.

4.2.1.6. (-)-(4S(R),6R(S))-4-((tert-Butyldimethylsilyl)oxy)-6(((1R,5S)-6,6-dimethylbicyclo[3.1.1]hept-2-en-2-yl)methoxy)tetrahydro-2H-pyran-2-one (12c). Yield $7.6 \mathrm{mg}, 2 \%$. Colourless oil; HPLC $t_{\mathrm{R}}=37 \mathrm{~min}$ (petroleum ether/ethyl acetate $94: 6$; flow $=0.8 \mathrm{~mL} / \mathrm{min}$ ); $[\alpha]_{\mathrm{D}}^{20}-129.0$ ( c 0.1, $\mathrm{CHCl}_{3}$ ); IR (film) $\nu_{\max } 2932,2858,1752,1469$, 1369, 1256, 1103, 1023, 838, $777 \mathrm{~cm}^{-1} ;{ }^{1} \mathrm{H}$ NMR (400 MHz, CDCl $)$ (see Table 4); ${ }^{13} \mathrm{C}$ NMR (100 $\mathrm{MHz}, \mathrm{CDCl}_{3}$ ) (see Table S3); HRMS $\left(\mathrm{Cl}^{+}\right): m / z[\mathrm{M}+\mathrm{H}]^{+}$, found 381.2453. $\mathrm{C}_{21} \mathrm{H}_{37} \mathrm{O}_{4} \mathrm{Si}$ requires 381.2461.

4.2.1.7. (-)-(4R(S),6S(R))-4-((tert-Butyldimethylsilyl)oxy)-6(((1R,2S,5R)-2-isopropyl-5-methylcyclohexyl)oxy)tetrahydro-2H-pyran-2-one (13a). Yield $7.7 \mathrm{mg}, 2 \%$. Colourless oil; HPLC $t_{\mathrm{R}}=16 \mathrm{~min}$ (petroleum ether/ethyl acetate 93:7; flow=3.0 mL/min); $[\alpha]_{\mathrm{D}}^{20}$ -67.7 (c 0.15, $\mathrm{CHCl}_{3}$ ); IR (film) $\nu_{\max } 2929,2858,1753,1463,1369$, 1255, 1101, 1023, 838, $777 \mathrm{~cm}^{-1}$; ${ }^{1} \mathrm{H}$ NMR (400 MHz, $\mathrm{CDCl}_{3}$ ) (see Table 3$) ;{ }^{13} \mathrm{C} \mathrm{NMR}\left(100 \mathrm{MHz}, \mathrm{CDCl}_{3}\right.$ ) (see Table S2); $\mathrm{HRMS}\left(\mathrm{CI}^{+}\right): \mathrm{m} / z$ $[\mathrm{M}+\mathrm{H}]^{+}$, found 385.2764. $\mathrm{C}_{21} \mathrm{H}_{41} \mathrm{O}_{4} \mathrm{Si}$ requires 385.2774.

4.2.1.8. (+)-(4S(R),6R(S))-4-((tert-Butyldimethylsilyl)oxy)-6(((1R,2S,5R)-2-isopropyl-5-methylcyclohexyl)oxy)tetrahydro-2H-pyran-2-one (13c). Yield $2.3 \mathrm{mg}, 0.6 \%$. Colourless oil; HPLC $t_{\mathrm{R}}=21 \mathrm{~min}$ (petroleum ether/ethyl acetate 93:7; flow $=3.0 \mathrm{~mL} / \mathrm{min}$ ); $[\alpha]_{\mathrm{D}}^{20}+21.1$ (c 0.1, $\mathrm{CHCl}_{3}$ ); IR (film) $\nu_{\max } 2930,2859,1752,1464,1369,1253,1104$, 1013, 837, $778 \mathrm{~cm}^{-1}$; ${ }^{1} \mathrm{H}$ NMR (400 MHz, $\mathrm{CDCl}_{3}$ ) (see Table 4$) ;{ }^{13} \mathrm{C}$ NMR (100 MHz, $\mathrm{CDCl}_{3}$ ) (see Table S3); HRMS $\left(\mathrm{Cl}^{+}\right): m / z[\mathrm{M}+\mathrm{H}]^{+}$, found 385.2766. $\mathrm{C}_{21} \mathrm{H}_{41} \mathrm{O}_{4} \mathrm{Si}$ requires 385.2774.

4.2.1.9. (+)-(4R,6S)-4-((tert-Butyldimethylsilyl)oxy)-6-((R)-1(naphthalen-2-yl)ethoxy)tetrahydro-2H-pyran-2-one (14a). Yield $58.8 \mathrm{mg}, 14.7 \%$. Colourless oil; HPLC $t_{\mathrm{R}}=42 \mathrm{~min}$ (petroleum ether/ ethyl acetate 93:7; flow $=3.0 \mathrm{~mL} / \mathrm{min}) ;[\alpha]_{\mathrm{D}}^{20}+118.0\left(\right.$ ( $\left.1.0, \mathrm{CHCl}_{3}\right) ; \mathrm{IR}$ (film) $\nu_{\max } 3060,2955,2858,1749,1471,1382,1231,1100,837$, $749 \mathrm{~cm}^{-1} ;{ }^{1} \mathrm{H}$ NMR (400 $\mathrm{MHz}, \mathrm{CDCl}_{3}$ ) (see Table 2); ${ }^{13} \mathrm{C}$ NMR
(100 MHz, $\mathrm{CDCl}_{3}$ ) (see Table S1); HRMS (ESI-QTOF): $m / z$ [M+Na $]^{+}$, found 423.1968. $\mathrm{C}_{23} \mathrm{H}_{32} \mathrm{O}_{4} \mathrm{NaSi}$ requires 423.1968; [M+H$\left.\mathrm{C}_{6} \mathrm{H}_{15} \mathrm{SiOH}\right]^{+}$, found 269.1161. $\mathrm{C}_{17} \mathrm{H}_{17} \mathrm{O}_{3}$ requires 269.1178 .

4.2.1.10. (+)-(4S,6S)-4-((tert-Butyldimethylsilyl)oxy)-6-((R)-1(naphthalen-2-yl)ethoxy)tetrahydro-2H-pyran-2-one (14b). Yield $1.2 \mathrm{mg}, 0.3 \%$. Colourless oil; HPLC $t_{\mathrm{R}}=34 \mathrm{~min}$ (petroleum ether/ethyl acetate 93:7; flow =3.0 mL/min); $[\alpha]_{\mathrm{D}}^{20}+91.4$ (c 1.4, $\left.\mathrm{CHCl}_{3}\right)$; IR (film) $\nu_{\max } 3056,2955,2858,1749,1462,1378,1255,1095,1027,837$, $778 \mathrm{~cm}^{-1} ;{ }^{1} \mathrm{H}$ NMR (400 MHz, $\mathrm{CDCl}_{3}$ ) (see Table 2); ${ }^{13} \mathrm{C}$ NMR $\left(100 \mathrm{MHz}, \mathrm{CDCl}_{3}\right.$ ) (see Table S1); HRMS (ESI-QTOF): $m / z[\mathrm{M}+\mathrm{Na}]^{+}$, found 423.1968. $\quad \mathrm{C}_{23} \mathrm{H}_{32} \mathrm{O}_{4} \mathrm{NaSi}$ requires 423.1968; $\left[\mathrm{M}+\mathrm{H}-\mathrm{C}_{6} \mathrm{H}_{15} \mathrm{SiOH}\right]^{+}$, found 269.1175. $\mathrm{C}_{17} \mathrm{H}_{17} \mathrm{O}_{3}$ requires 269.1178 .

4.2.1.11. (-)-(4S,6R)-4-((tert-Butyldimethylsilyl)oxy)-6-((S)-1(naphthalen-2-yl)ethoxy)tetrahydro-2H-pyran-2-one (15a). Yield $58.7 \mathrm{mg}, 14.7 \%$. $[\alpha]_{\mathrm{D}}^{20}-118.0\left(\right.$ c $\left.1.0, \mathrm{CHCl}_{3}\right)$.

4.2.1.12. (-)-(4R,6R)-4-((tert-Butyldimethylsilyl)oxy)-6-((S)-1(naphthalen-2-yl)ethoxy)tetrahydro-2H-pyran-2-one (15b). Yield $1.3 \mathrm{mg}, 0.3 \%$. $[\alpha]_{\mathrm{D}}^{20}-91.4$ (c $1.3, \mathrm{CHCl}_{3}$ ).

4.2.2. Preparation of (-)-(R)-Methyl 3-(tert-butyldimethylsilyloxy)5-oxopentanoate $((-)-(R)-8)$. Sodium methoxide $(654.5 \mathrm{mg}$, $12.12 \mathrm{mmol}$ ) was added to a solution of tetrahydro-2 $\mathrm{H}$-pyran-2-one (+)-14a (809.0 mg, $2.02 \mathrm{mmol})$ in dry $\mathrm{MeOH}(101 \mathrm{~mL})$ at $-35^{\circ} \mathrm{C}$. The reaction mixture was stirred at $-35^{\circ} \mathrm{C}$ for $24 \mathrm{~h}$, then quenched with saturated $\mathrm{NH}_{4} \mathrm{Cl}(110 \mathrm{~mL})$. The mixture was then allowed to warm to room temperature and diethyl ether $(300 \mathrm{~mL})$ was added. The layers were separated and the aqueous phase was further extracted with diethyl ether $(3 \times 300 \mathrm{~mL})$. The combined organic layers were washed with brine $(2 \times 500 \mathrm{~mL})$, dried over $\mathrm{Na}_{2} \mathrm{SO}_{4}$ and the solvent was removed under reduced pressure to give a crude reaction product, which was purified by column chromatography (petroleum ether $/ \mathrm{Et}_{2} \mathrm{O}$ 90:10) to give (R)-1-phenylethanol (157.0 $\mathrm{mg}, 64 \%$ ) and (-)-(R)-8 (275.0 mg, 52\%, 99\% ee) as a colourless oil; $[\alpha]_{\mathrm{D}}^{20}-10.2$ (c $\left.0.3, \mathrm{CHCl}_{3}\right)$; IR (film) $\nu_{\max } 2931,2898,2857$, 1736, 1438, 1255, 1087, 837, $777 \mathrm{~cm}^{-1} ;{ }^{1} \mathrm{H}$ NMR (400 MHz, $\mathrm{CDCl}_{3}$ ) $\delta 9.75(1 \mathrm{H}, \mathrm{t}, J=2.0 \mathrm{~Hz}), 4.59(1 \mathrm{H}$, quint, $J=6.2 \mathrm{~Hz}), 3.63(3 \mathrm{H}, \mathrm{s})$, 2.67-2.62 (2H, m), $2.52(2 \mathrm{H}, \mathrm{m}), 0.80(9 \mathrm{H}, \mathrm{s}), 0.03(6 \mathrm{H}, \mathrm{s}) ;{ }^{13} \mathrm{C} \mathrm{NMR}$ $\left(100 \mathrm{MHz}, \mathrm{CDCl}_{3}\right) \delta 200.8,171.1,64.9,51.6,50.8,42.3,25.5$ (3C), 17.8, -4.9 (2C); HRMS $\left(\mathrm{CI}^{+}\right): \mathrm{m} / z\left[\mathrm{M}-\mathrm{CH}_{3}\right]^{+}$, found $245.1199 . \mathrm{C}_{11} \mathrm{H}_{21} \mathrm{O}_{4} \mathrm{Si}$ requires 245.1209. The enantiomeric excess of 8 could be determined by chiral gas chromatography, using the general conditions stated above; isocratic $80^{\circ} \mathrm{C} . t_{\mathrm{R}}(\mathrm{min}): 251.36$.

4.2.3. Preparation of (+)-(S)-methyl 3-(tert-butyldimethylsilyloxy)5-oxopentanoate ((+)-(S)-9). Compound (-)-15a $(660.0 \mathrm{mg}$, $1.65 \mathrm{mmol}$ ) was converted into $(+)-(S)$-methyl 3-(tert-butyldimethylsilyloxy)-5-oxopentanoate $((+)-(S)-9)(220.0 \mathrm{mg}, 50 \%, 99 \%$ ee) following the methodology described above for the synthesis of $(-)-(R)-8$ from $(+)-14 a$. Colourless oil; $[\alpha]_{\mathrm{D}}^{20}+10.2\left(c 0.25, \mathrm{CHCl}_{3}\right)$. The enantiomeric excess of $\mathbf{9}$ could be determined by chiral gas chromatography, using the general conditions stated above; isocratic $80^{\circ} \mathrm{C} . t_{\mathrm{R}}(\min ): 256.19$.

4.2.4. Preparation of (-)-(R)-methyl 3-(tert-butyldimethylsilyloxy)5-hydroxypentanoate ((R)-16). Sodium borohydride $(28.5 \mathrm{mg}$, $0.75 \mathrm{mmol})$ was added to a solution of $(R)-8(153.7 \mathrm{mg}, 0.59 \mathrm{mmol})$ in dry $\mathrm{MeOH}(101 \mathrm{~mL})$ at $-35^{\circ} \mathrm{C}$ under an argon atmosphere. The reaction mixture was stirred at $-35{ }^{\circ} \mathrm{C}$ for $24 \mathrm{~h}$, adding two supplementary portions of $\mathrm{NaBH}_{4}(15 \mathrm{mg}$ each portion). Then, $1 \mathrm{M} \mathrm{HCl}$ was added until pH $7(8 \mathrm{~mL})$ and the reaction mixture was allowed to warm to room temperature. Solvent was removed under reduced pressure and ethyl acetate $(50 \mathrm{~mL})$ and brine $(15 \mathrm{~mL})$ were added. The layers were separated and the aqueous layer was further 
extracted with ethyl acetate $(3 \times 50 \mathrm{~mL})$. The combined organic layers were washed with brine $(100 \mathrm{~mL})$, dried over $\mathrm{Na}_{2} \mathrm{SO}_{4}$, filtered and the solvent was removed under reduced pressure to give the crude reaction product, which was purified by column chromatography (petroleum ether/EtOAc 90:10) to give $(R)-\mathbf{1 6}(106.7 \mathrm{mg}$, $69 \%)$ and $(R)-4$-((tert-butyldimethylsilyl)oxy)tetrahydro-2H-pyran2-one $(R)$-17 (12.4 mg, 9\%). Data for $(R)-16$ : colourless oil; $[\alpha]_{D}^{20}-2.0$ (c 3.1, $\mathrm{CHCl}_{3}$ ); IR (film) $\nu_{\max } 3422,2930,1741,1438,1257,1161,1026$, 837, $777 \mathrm{~cm}^{-1}$; ${ }^{1} \mathrm{H}$ NMR (400 MHz, $\left.\mathrm{CDCl}_{3}\right) \delta 4.30(1 \mathrm{H}, \mathrm{ddt}, J=12.4$, 6.4, $4.8 \mathrm{~Hz}), 3.76-3.66(2 \mathrm{H}, \mathrm{m}), 3.64(3 \mathrm{H}, \mathrm{s}), 2.53$ (2H, dd, $J=17.6$, $6.4 \mathrm{~Hz}), 2.47(2 \mathrm{H}, \mathrm{dd}, J=17.6,6.4 \mathrm{~Hz}), 2.42(\mathrm{~s}, \mathrm{OH}), 1.85-1.65(2 \mathrm{H}, \mathrm{m})$, 0.84 (9H, s), 0.07 (3H, s), $0.04(3 \mathrm{H}, \mathrm{s}) ;{ }^{13} \mathrm{C} \mathrm{NMR}\left(100 \mathrm{MHz}, \mathrm{CDCl}_{3}\right)$ $\delta 171.8,68.0,59.4,51.5,42.1,38.9,25.6$ (3C), 17.8, -4.8, -4.9; HRMS $\left(\mathrm{CI}^{+}\right): \mathrm{m} / \mathrm{z}\left[\mathrm{M}-\mathrm{C}\left(\mathrm{CH}_{3}\right)_{3}\right]^{+}$, found 205.0899. $\mathrm{C}_{8} \mathrm{H}_{17} \mathrm{O}_{4} \mathrm{Si}$ requires 205.0896 .

4.2.5. Preparation of (+)-(S)-methyl 3-(tert-butyldimethylsilyloxy)5-hydroxypentanoate ((S)-18). (+)-(S)-Methyl 3-(tert-butyldimethylsilyloxy)-5-oxopentanoate $((S)-9)(110.0 \mathrm{mg}, 0.42 \mathrm{mmol})$ was converted into (+)-(S)-methyl 3-(tert-butyldimethylsilyloxy)-5hydroxypentanoate $((S)-\mathbf{1 8})(85.0 \mathrm{mg}, 72 \%)$ and $(S)-\mathbf{1 9}(7.0 \mathrm{mg}$, 7\%) following the methodology described above for the synthesis of $(R)-16$ from $(R)-8$. Data for $(S)$-18: colourless oil; $[\alpha]_{D}^{20}+2.0$ (c 0.2, $\left.\mathrm{CHCl}_{3}\right)$.

4.2.6. Preparation of (-)-(R)-4-((tert-butyldimethylsilyl)oxy)tetrahydro-2H-pyran-2-one ((R)-17). $p$-Toluenesulfonic acid monohydrate (PTSA) $(1.8 \mathrm{mg}, 0.01 \mathrm{mmol})$ was added to a solution of $(-)-(R)$-methyl 3-(tert-butyldimethylsilyloxy)-5hydroxypentanoate $((R)-\mathbf{1 6})(38.0 \mathrm{mg}, 0.15 \mathrm{mmol})$ in dry $\mathrm{CH}_{2} \mathrm{Cl}_{2}$ $(2.3 \mathrm{~mL})$ at room temperature. When TLC analysis indicated the completion of the reaction $(1 \mathrm{~h})$, saturated $\mathrm{NaHCO}_{3}$ was added $(3 \mathrm{~mL})$ and the mixture was stirred for a further $15 \mathrm{~min}$. The aqueous phase was then extracted with ethyl acetate $(3 \times 25 \mathrm{~mL})$, the combined organic layers were washed with brine $(50 \mathrm{~mL})$, dried over $\mathrm{Na}_{2} \mathrm{SO}_{4}$ and filtered. Evaporation of the solvent gave the crude product that was purified by silica gel column chromatography (petroleum ether/EtOAc 90:10), to yield $(R)-\mathbf{1 7}(28.9 \mathrm{mg}, 87 \%, 89 \%$ ee). Colourless oil; $[\alpha]_{D}^{20}-2.8$ (c 0.1, $\mathrm{CHCl}_{3}$ ); IR (film) $\nu_{\max } 2930$, 2857, 1739, 1472, 1260, 1161, 1085, 837, $778 \mathrm{~cm}^{-1} ;{ }^{1} \mathrm{H}$ NMR $\left(300 \mathrm{MHz}, \mathrm{CDCl}_{3}\right) \delta 4.56(1 \mathrm{H}, \mathrm{ddd}, J=11.2,9.6,3.9 \mathrm{~Hz}), 4.30-4.22$ $(2 \mathrm{H}, \mathrm{m}), 2.69(1 \mathrm{H}, \mathrm{dd}, J=17.4,5.0 \mathrm{~Hz}), 2.52(1 \mathrm{H}, \mathrm{dd}, J=17.4,4.2 \mathrm{~Hz})$, 2.05-1.94 (1H, m), 1.83-1.73 (1H, m), $0.86(9 \mathrm{H}, \mathrm{s}), 0.06(3 \mathrm{H}, \mathrm{s}), 0.05$ $(3 \mathrm{H}, \mathrm{s}) ;{ }^{13} \mathrm{C} \mathrm{NMR}\left(75 \mathrm{MHz}, \mathrm{CDCl}_{3}\right) \delta 170.0,65.3,63.9,40.0,31.3,25.6$ (3C), 18.0, -4.8, -4.9; HRMS $\left(\mathrm{CI}^{+}\right): \mathrm{m} / z\left[\mathrm{M}-\mathrm{C}\left(\mathrm{CH}_{3}\right)_{3}\right]^{+}$, found 173.0645. $\mathrm{C}_{7} \mathrm{H}_{13} \mathrm{O}_{3} \mathrm{Si}$ requires 173.0634. The enantiomeric excess of 17 could be determined by chiral gas chromatography, using the general conditions stated above; isocratic $100{ }^{\circ} \mathrm{C} . t_{\mathrm{R}}(\mathrm{min}): 244.97$ (minor), 249.28 (major).

4.2.7. Preparation of (+)-(S)-4-((tert-butyldimethylsilyl)oxy)tetrahydro-2H-pyran-2-one ((S)-19). (+)-(S)-Methyl 3-(tert-butyldimethylsilyloxy)-5-hydroxypentanoate $((S)-18)(48.0 \mathrm{mg}, 0.18 \mathrm{mmol})$ was converted into (+)-(S)-4-((tert-butyldimethylsilyl)oxy)tetrahydro-2H-pyran-2-one ((S)-19) (35.6 $\mathrm{mg}, 84 \%, 96 \%$ ee) following the methodology described above for the synthesis of $(R)$-17 from $(R)$-16. Colourless oil; $[\alpha]_{\mathrm{D}}^{20}+2.9\left(c \quad 0.3, \mathrm{CHCl}_{3}\right)$. The enantiomeric excess of 19 could be determined by chiral gas chromatography, using the general conditions stated above; isocratic $100{ }^{\circ} \mathrm{C} . t_{\mathrm{R}}$ (min): 238.88 (major), 242.38 (minor).

4.2.8. Preparation of (-)-(R)-4-hydroxytetrahydro-2H-pyran-2-one $((R)-4)$. ( -)-(R)-4-((tert-Butyldimethylsilyl)oxy)tetrahydro-2H-pyran-2-one $((R)-17)(27.4 \mathrm{mg}, 0.12 \mathrm{mmol})$ was converted into $(-)-(R)-4$-hydroxytetrahydro-2H-pyran-2-one $((R)-4)(9.7 \mathrm{mg}, 70 \%$, $79 \%$ ee) following the methodology described in the literature. ${ }^{20}$
Colourless oil; $[\alpha]_{\mathrm{D}}^{20}-3.1$ (c $0.34, \mathrm{CHCl}_{3}$ ); IR (film) $\nu_{\max } 3422$, 2926, 2875, 1719, 1458, 1383, 1261, $1079 \mathrm{~cm}^{-1}$; ${ }^{1} \mathrm{H}$ NMR $(400 \mathrm{MHz}$, $\left.\mathrm{CDCl}_{3}\right) \delta 4.57(1 \mathrm{H}$, ddd, $J=11.4,8.0,4.0 \mathrm{~Hz}), 4.34(1 \mathrm{H}$, quint, $J=5.2 \mathrm{~Hz}), 4.28(1 \mathrm{H}$, ddd, $J=11.4,6.4,4.8 \mathrm{~Hz}), 2.82(1 \mathrm{H}, \mathrm{dd}, J=17.6$, $5.2 \mathrm{~Hz}), 2.58(1 \mathrm{H}, \mathrm{ddd}, J=17.6,5.2,1.0 \mathrm{~Hz}), 2.12(1 \mathrm{H}, \mathrm{m}), 1.89(1 \mathrm{H}, \mathrm{m})$, $1.64(\mathrm{~s}, \mathrm{OH}) ;{ }^{13} \mathrm{C}$ NMR $\left(100 \mathrm{MHz}, \mathrm{CDCl}_{3}\right) \delta 170.0,65.2,63.4,39.4$, 31.0; HRMS $\left(\mathrm{CI}^{+}\right): \mathrm{m} / z[\mathrm{M}]^{+}$, found 116.0475. Calcd for $\mathrm{C}_{5} \mathrm{H}_{8} \mathrm{O}_{3}$ 116.0473. The enantiomeric excess of 4 could be determined by chiral gas chromatography, using the general conditions stated above; isocratic $100{ }^{\circ} \mathrm{C} . t_{\mathrm{R}}(\mathrm{min})$ : 208.23 (minor), 211.67 (major).

4.2.9. Preparation of (+)-(S)-4-hydroxytetrahydro-2H-pyran-2-one ((S)-5). (+)-(S)-4-((tert-Butyldimethylsilyl)oxy)tetrahydro-2H-pyran-2-one ((S)-19) (31.1 mg, $0.14 \mathrm{mmol})$ was converted into $(+)-(S)$ 4-hydroxytetrahydro-2H-pyran-2-one ((S)-5) (10.5 mg, 67\%, 96\% ee) following the methodology described in the literature. ${ }^{20}$ Colourless oil; $[\alpha]_{\mathrm{D}}^{20}+3.6\left(c 0.2, \mathrm{CHCl}_{3}\right)$. The enantiomeric excess of $\mathbf{5}$ could be determined by chiral gas chromatography, using the general conditions stated above; isocratic $100^{\circ} \mathrm{C} . t_{R}(\min ): 204.26$ (major), 217.71 (minor).

\section{Acknowledgements}

This research was supported by grants from MINECO (AGL201239798-C02-01) and from the Junta de Andalucía (P07-FQM-02689) (Spain). A.J.M.-S. gratefully acknowledges a Royal Society of Chemistry research fund grant (UK). J.M.B. thanks Junta de Andalucía for Research Fellowship. Use of NMR and mass spectrometry (QTOF) facilities at Servicio Centralizado de Ciencia y Tecnología (SCCYT) of the University of Cádiz is acknowledged.

\section{Supplementary data}

${ }^{13} \mathrm{C}$ spectroscopic data for compounds $6 \mathbf{a}, \mathbf{b}, 7 \mathbf{7}, 10 \mathbf{a}-14 \mathbf{a}, 14 \mathbf{b}, 7 \mathrm{c}$ and 10c-13c together with ${ }^{1} \mathrm{H}$ and ${ }^{13} \mathrm{C}$ NMR spectra for compounds 4, 6a,b, 7a, 7c, 8, 10a-14a, 10c-13c, 14b, 16,17, selected NOESY 1D for compounds 11a, 11c, 14a and 14b, and chiral GC chromatograms for compounds $\mathbf{4}, \mathbf{5}, \mathbf{8}, \mathbf{9}, \mathbf{1 7}$, and $\mathbf{1 9}$. Supplementary data associated with this article can be found in the online version, at http:// dx.doi.org/10.1016/j.tet.2015.08.010. These data include MOL files and InChiKeys of the most important compounds described in this article.

\section{References and notes}

1. (a) Rovis, T. In New Frontiers in Asymmetric Catalysis; Mikami, K., Lautens, M., Eds.; Wiley: Hoboken, NJ, 2006; pp 275-311; (b) Anstiss, M.; Holland, J. M.; Nelson, A.; Titchmarsh, J. R. Synlett 2003, 1213; (c) Willis, M. C. J. Chem. Soc., Perkin Trans. 1 1999, 1765; (d) Ward, R. S. Chem. Soc. Rev. 1990, 19, 1.

2. (a) Garcia-Urdiales, E.; Alfonso, I.; Gotor, V. Chem. Rev. 2011, 111, PR110; (b) Garcia-Urdiales, E.; Alfonso, I.; Gotor, V. Chem. Rev. 2005, 105, 313.

3. Hoffmann, R. Angew. Chem., Int. Ed. 2003, 42, 1096.

4. Nakahara, K.; Fujioka, H. Symmetry 2010, 2, 437.

5. Atodiresei, I.; Schiffers, I.; Bolm, C. Chem. Rev. 2007, 107, 5683.

6. Rendler, S.; Oestreich, M. Angew. Chem., Int. Ed. 2008, 47, 248.

7. (a) Strand, D.; Norrby, P.; Rein, T. J. Org. Chem. 2006, 71, 1879; (b) Vares, L.; Rein, T. Org. Lett. 2000, 2, 2611; (c) Tullis, J. S.; Vares, L.; Kann, N.; Norrby, P.; Rein, T. J. Org. Chem. 1998, 63, 8284; (d) Kann, N.; Rein, T. J. Org. Chem. 1993, 58, 3802.

8. (a) Bouzbouz, S.; Popkin, M. E.; Cossy, J. Org. Lett. 2000, 2, 3449; (b) Takemoto, Y.; Baba, Y.; Honda, A.; Nakao, S.; Noguchi, I.; Iwata, C.; Tanaka, T.; Ibuka, T. Tetrahedron 1998, 54, 15567; (c) Takemoto, Y.; Baba, Y.; Noguchi, I.; Iwata, C. Tetrahedron Lett. 1996, 37, 3345; (d) Wang, Z.; Deschênes, D. J. Am. Chem. Soc. 1992, 114, 1090; (e) Roush, W. R.; Park, J. C. Tetrahedron Lett. 1990, 31, 4707.

9. Grachan, M. L.; Tudge, M. T.; Jacobsen, E. N. Angew. Chem., Int. Ed. 2008, 47, 1469.

10. (a) Oppolzer, W.; Walther, E.; Pérez Balado, C.; De Brabander, J. Tetrahedron Lett. 1997, 38, 809; (b) Oppolzer, W.; De Brabander, J.; Walther, E.; Bernardinelli, G. Tetrahedron Lett. 1995, 36, 4413; (c) Ziegler, F. E.; Becker, M. R. J. Org. Chem. 1990, 55, 2800.

11. Dodd, K.; Morton, D.; Worden, S.; Narquizian, R.; Nelson, A. Chem.-Eur. J. 2007, 13, 5857.

12. Mans, D. M.; Pearson, W. H. Org. Lett. 2004, 6, 3305. 
13. (a) Rosen, T.; Heathcock, C. H. J. Am. Chem. Soc. 1985, 107, 3731; (b) Theisen, P. D. Heathcock, C. H. J. Org. Chem. 1988, 53, 2374; (c) Chauman, K. Bhatt, R. K. Falck, J. R.; Capdevila, J. H. Tetrahedron Lett. 1994, 35, 1825; (d) Kumar, A. Dittmer, D. C. J. Org. Chem. 1994, 59, 4760; (e) Loubinoux, B.; Sinnes, J.-L.; O'Sullivan, A. C.; Winkler, T. Tetrahedron 1995, 51, 3549.

14. (a) Beuerle, T.; Schreier, P.; Brunerie, P.; Bicchi, C.; Schwab, W. Phytochemistry 1996, 43, 145; (b) Sjögren, J.; Magnusson, J.; Broberg, A.; Schnürer, J.; Kenne, L. Appl. Environ. Microbiol. 2003, 69, 7554.

15. Carosi, L.; Hall, D. Can. J. Chem. 2009, 87, 650 and references included therein.

16. Stokker, G. E.; Alberts, A. W.; Anderson, P. S.; Cragoe, E. J.; Deana, A. A.; Gilfillan, J. L.; Hirshfield, J.; Holtz, W. J.; Hoffman, W. F.; Huff, J. W.; Lee, T. J.; Novello, F. C.; Prugh, J. D.; Rooney, C. S.; Smith, R. L.; Willard, A. K. J. Med. Chem. 1986, 29, 170.

17. Huang, C. -J.; Wang, S. -C.; Kuo, Y. -H.; Hong, Y. -H.; Lin, B. -F.; Hsu, C. U.S. Patent US 20,100,125,102 A1, 2010; Chem. Abstr. 2010, 152, 561033.

18. Kudoh, T.; Park, C. S.; Lefurgy, S. T.; Sun, M.; Michels, T.; Leyh, T. S.; Silverman, R. Bioorg. Med. Chem. 2010, 18, 1124
19. Collado, I. G.; Sanchez, A. J. M.; Hanson, J. R. Nat. Prod. Rep. 2007, $24,674$.

20. Botubol-Ares, J. M.; Durán-Peña, M. J.; Hernández-Galán, R.; Collado, I. G.; Harwood, L. M.; Macías-Sánchez, A. J. Bioorg. Med. Chem. 2015, 23, 6325.

21. Buckley, S. L. J.; Drew, M. G. B.; Harwood, L. M.; Macías-Sánchez, A. J. Tetrahedron Lett. 2002, 43, 3593

22. (a) Miyashita, M.; Suzuki, T.; Hoshino, M.; Yoshikoshi, A. Tetrahedron 1993, 53, 12469; (b) Kitanosono, T.; Xu, P.; Kobayashi, S. Chem. Asian J. 2014, 9, 179.

23. Determined by chiral GC.

24. Novák, L.; Rohály, J.; Poppe, L.; Hornyánszky, G.; Kolonits, P.; Zelei, I.; Fehér, I.; Fekete, J.; Szabó, E.; Záhorszky, U.; Jávor, A.; Szántay, C. Liebigs Ann. Chem. 1992 145.

25. See, for instance: Yu, X. M.; Han, H.; Blagg, B. S. J. J. Org. Chem. 2005, 70, 5599. 26. Smith, A. B.; Ott, G. R. J. Am. Chem. Soc. 1996, 118, 13095.

27. Lower enantiomeric excesses, particularly for $(-)-(R)-\mathbf{1 7}$ and $(-)-(R)-\mathbf{4}$, can be attributed to an incomplete removal of PTSA during the work-up. 